\title{
The Effects of Laser Moxibustion on Knee Osteoarthritis Pain in Rats
}

\author{
Yuan Li, MD, ${ }^{1}$ Fan Wu, PhD, Jianzi Wei, PhD, ${ }^{1}$ Lixing Lao, $\mathrm{PhD},{ }^{1,2}$ and Xueyong Shen, MD ${ }^{1,3}$
}

\begin{abstract}
Background: Although chronic pain affects the quality of life of patients with osteoarthritis, current medical treatments are either ineffective or have long-term side effects. Recently, low-intensity laser irradiation of corresponding acupoints was demonstrated to alleviate pain.

Objective: The aim of the present study was to investigate the effects of $10.6 \mu \mathrm{m}$ laser moxibustion on a monosodium iodoacetate (MIA)-induced knee osteoarthritis pain model.

Methods: Thirty-two rats were randomly assigned to four groups: Saline, MIA, MIA+Laser, and MIA+Sham Laser. The $10.6 \mu \mathrm{m}$ laser was used to irradiate the ST35 for $10 \mathrm{~min}$ once a day for a total of seven applications. The paw withdrawal mechanical threshold and weight-bearing difference were performed to evaluate the analgesic effects of laser moxibustion. At the end of the experiment on days 28, the joint histology, the levels of metalloproteinases-13 (MMP-13) in the cartilage, and TNF- $\alpha$, IL-1 $\beta$, and IL-6 in the synovial membrane were measured to determine the chondroprotection and anti-inflammatory effect of laser moxibustion.

Results: Early laser moxibustion significantly reversed the MIA-induced mechanical hyperalgesia and weightbearing difference, especially on the 28th day $(p<0.001)$. Moreover, laser moxibustion prevented the articular pathological lesions and cartilage destruction on days $28(p<0.01)$. Remarkably, the levels of cartilage MMP13 , and synovial TNF- $\alpha$, IL-1 $\beta$, and IL-6 also decreased on day $28(p<0.05)$ after the early treatment of laser moxibustion.

Conclusions: $10.6 \mu \mathrm{m}$ laser moxibustion may have long-lasting analgesic, anti-inflammatory, and chondroprotection effects, suggesting that it may emerge as a potential therapeutic strategy for the chronic pain treatment of osteoarthritis.
\end{abstract}

Keywords: laser moxibustion, knee osteoarthritis, chronic pain

\section{Introduction}

$\mathbf{K}$ NEE OSTEOARTHRITIS (KOA) is a degenerative disease, including synovitis, articular cartilage destruction, subchondral bone reconstruction, and other pathological changes, accompanied by persistent pain and joint dysfunction, greatly affecting the quality of life of patients. ${ }^{1}$ However, the mechanism of chronic pain in KOA remains unclear. In recent years, animal models simulating the pathological changes of human KOA have provided a broad space for the study of the molecular mechanism of KOA. ${ }^{2}$ The intra-articular injection of monosodium iodoacetate (MIA), a commonly used chemical KOA model, results in the histological changes of articular cartilage and especially, a nociceptive response similar to the pathology and pain sensation observed in patients with KOA. ${ }^{3,4}$

There are studies on the structural changes of KOA induced by MIA, and pain mechanisms are limited to the local joint at the peripheral level, ${ }^{5}$ involving inflammatory mediators, structural proteins (extracellular matrix hydrolase), and cell signaling pathway-related protein kinases-mediated synovitis and chondrocyte apoptosis. ${ }^{6-8}$ Synovial inflammation of the knee joint induces the infiltration of immune cells and secretes a large number of inflammatory mediators,

\footnotetext{
${ }^{1}$ School of Acupuncture, Moxibustion and Tuina, Shanghai University of Traditional Chinese Medicine, Shanghai, China.

${ }^{2}$ School of Chinese Medicine, The University of Hong Kong, Hong Kong, SAR, China.

${ }^{3}$ Shanghai Research Center of Acupuncture \& Meridian, Shanghai, China.

(C) Yuan Li, et al., 2019; Published by Mary Ann Liebert, Inc. This Open Access article is distributed under the terms of the Creative Commons License (http://creativecommons.org/licenses/by/4.0), which permits unrestricted use, distribution, and reproduction in any medium, provided the original work is properly credited.
} 
such as proinflammatory cytokines (TNF- $\alpha$, IL-1 $\beta$, and IL-6), which further increase the expression of matrix metalloproteinases (MMPs), promote the catabolism of chondrocyte, and aggravate the degradation of extracellular matrix. ${ }^{9}$

For the treatment of KOA, nonsteroidal anti-inflammatory drugs are primarily used for acute pain, but are not particularly effective for chronic pain. ${ }^{10,11}$ Nondrug therapies, such as acupuncture, moxibustion, low-intensity laser, proper exercise, and weight control, can also alleviate pain and improve articular function to varying degrees, but the exact therapeutic mechanism has not been clarified. ${ }^{12-14}$

Laser acupuncture, a product combining modern laser, traditional acupuncture and moxibustion, has been used in the treatment of inflammatory pain, and its curative effect is remarkable. ${ }^{13,15,16}$ The low-intensity laser irradiation of corresponding acupoints was also demonstrated to significantly alleviate the pain of patients with KOA. ${ }^{13,15,16} \mathrm{~A}$ convincing study suggested that $10.6 \mu \mathrm{m}$ laser moxibustion has a good thermal therapeutic effect similar to moxibustion while avoiding the shortcomings of traditional moxibustioninduced smoke pollution and compensates for the limited warming effect of common laser needles currently used in clinical settings. ${ }^{17,18}$ However, the underlying mechanisms of its effects on chronic joint pain are still not completely understood. Our preliminary clinical trials have shown that laser moxibustion at a $10.6 \mu \mathrm{m}$ wavelength significantly improved joint pain and articular dysfunction in patients with KOA. ${ }^{19,20}$

To further determine the analgesic and cartilage protective effects of $10.6 \mu \mathrm{m}$ laser moxibustion on $\mathrm{KOA}$, we investigated the effect of $10.6 \mu$ m laser moxibustion on pain hypersensitivity and articular cartilage destruction in MIAinduced KOA model.

\section{Materials and Methods}

\section{Animals and ethics}

Male Sprague-Dawley rats (220-250g, SCXK20130016; Xipuer-Bikey Co., Ltd., Shanghai, China) were housed in a controlled condition $\left(22^{\circ} \mathrm{C}-24^{\circ} \mathrm{C}\right.$, relative humidity 40 $60 \%$, and 12:12 h light:dark cycle) with food and water ad libitum. All experimental procedures were approved by the Shanghai University of Traditional Chinese Medicine Animal Welfare and Ethics Committee (PZSHUTCM18113003) and conformed to the standards of the International Council for Laboratory Animal Science.

\section{MIA-induced KOA}

Animals $(n=32)$ were distributed randomly into four groups: Saline, MIA, MIA+Laser, and MIA+Sham Laser $(n=8$ per group). Rats in the MIA, MIA+Laser, and MIA+Sham Laser groups were anesthetized with isoflurane and subject to a single intra-articular injection of MIA (3 mg/50 $\mu \mathrm{L}$; Sigma) dissolved in $0.9 \%$ saline in the left knee joint through the infrapatellar ligament, as previously described. ${ }^{21}$ The MIA+Laser and MIA+Sham Laser groups received intervention procedures. The saline group only received $50 \mu \mathrm{L}$ of $0.9 \%$ saline into the left knee joint without any treatment.

\section{Laser moxibustion treatment}

Laser moxibustion treatment was applied using a $10.6 \mu \mathrm{m}$ laser device (SX10-C1; Wonderful-Opto-Electrics Tech Co, Ltd., Shanghai, China) as previously described..$^{21,22}$ One day after the MIA injection, rats were immobilized on the platform by the operator. Rats in the MIA+Laser group were exposed to laser irradiation, the parameters are presented in Table 1, at the ST-35 acupoint located in the depression of the lateral aspect of the infrapatellar ligament for seven consecutive days (once per day). ${ }^{23}$

For the MIA+Sham Laser group, the rats were subjected to the same procedure as the Laser group, but without laser output and with only the indicator lights on.

\section{Behavioral tests}

All rats were acclimated to the test environment for 30 min. The paw withdrawal threshold was measured using an Electronic Von Frey Aesthesiometer (IITC Life Science). The rigid probe was perpendicularly applied to the plantar surface of the ipsilateral hind paw with increasing force until paw withdrawal or a flinching response. The average of 3 sec-time was recorded as the result of paw withdrawal mechanical threshold (PWMT) for each rat. ${ }^{24}$ The weightbearing difference was tested using an Incapacitance Tester (MR-600; IITC Life Science). Rats were placed in a plexiglass chamber and each paw positioned separately on a loadbearing plate. The percentage of ipsilateral weight born was calculated according to the following: [(the weight distribution of the ipsilateral hind paw/the weight distribution of the ipsilateral plus contralateral hind paw) $\times 100 \%$ ]. The mean value of $3 \mathrm{sec}$-time was determined as the result of the weight born for each rat. ${ }^{21}$ The operator was blinded to grouping.

\section{Joint histology}

Rats were deeply anesthetized 28 days after the behavioral tests and transcardially perfused with $0.9 \%$ saline followed by $4 \%$ paraformaldehyde. Each left knee joint was resected, fixed in $10 \%$ formalin (Sigma-Aldrich) at $4^{\circ} \mathrm{C}$ for $48 \mathrm{~h}$, decalcified with ethylenediaminetetraacetic acid decalcifying solution (Solarbio) for 4 weeks, and then embedded in paraffin. The coronal sections $(7 \mu \mathrm{m})$ were stained with hematoxylin and eosin (HE) and Safranin O (SO)-fast green, and then observed using a microscope. Cartilage

Table 1. The Parameters of Laser Moxibustion Device

\begin{tabular}{|c|c|c|c|c|c|}
\hline $\begin{array}{l}\text { Wavelength, } \\
\mu \mathrm{m}\end{array}$ & $\begin{array}{l}\text { Irradiance, } \\
\mathrm{mW} / \mathrm{cm}^{2}\end{array}$ & $\begin{array}{c}\text { Energy } \\
\text { density, } \mathrm{J} / \mathrm{cm}^{2}\end{array}$ & $\begin{array}{l}\text { Each treatment } \\
\text { duration, min }\end{array}$ & $\begin{array}{l}\text { Treatment } \\
\text { frequency }\end{array}$ & $\begin{array}{c}\text { Cumulative } \\
\text { dose, } J\end{array}$ \\
\hline 10.6 & 80 & 1500 & 10 & One time per day & 329.86 \\
\hline
\end{tabular}


degeneration was assessed blindly using the Osteoarthritis Research Society International (OARSI) score, which combined the score of grade and stage $(0-24) .^{25}$

\section{Immunohistochemical staining}

The immunohistochemistry sections were deparaffinized, rehydrated, and blocked with normal goat serum. The sections were incubated with antibodies to MMP-3 (1:400, ab39012; Abcam) at $4^{\circ} \mathrm{C}$ overnight, followed by horseradish peroxidase-conjugated secondary antibody (1:200, ab97051; Abcam). The slides were visualized using 3,3-diaminobenzoic acid and counterstained with hematoxylin. Finally, the sections were mounted with a coverslip and Canada balsam. For the quantitative analysis of immunohistochemical staining, the immunoreactivity was determined through the average optical density (AOD) in the fields obtained from three sections for each rat using Image Pro plus 6.0 software. The operator was blinded to treatment.

\section{Enzyme-linked immunosorbent assay}

The synovial membrane was collected and centrifuged at $14,000 \mathrm{r} / \mathrm{min}$ at $4^{\circ} \mathrm{C}$ for $10 \mathrm{~min}$, and the supernatants were then extracted for the measurement of enzyme-linked immunosorbent assay (ELISA). The concentrations of TNF- $\alpha$, IL- $1 \beta$, and IL- 6 were analyzed using ELISA kits according to the manufacturer's protocol.

\section{Statistical analysis}

Data are presented as the mean \pm standard deviation and analyzed using GraphPad Prism7.0 software. The behavioral tests were analyzed by two-way ANOVA for repeated measurement followed by Bonferroni's post hoc test. The OARSI score was analyzed by Kruskal-Wallis test followed by Dunn's post hoc test among the groups. The values of MMP-13, TNF$\alpha$, IL- $1 \beta$, and IL- 6 were conducted by one-way ANOVA followed by Tukey's post hoc test for multiple comparisons. $p<0.05$ were considered statistically significant.

\section{Results}

\section{Laser moxibustion reversed MIA-induced} pain behavior

As expected, the injection of MIA presented decreased mechanical hypersensitivity threshold and weight-bearing difference in the ipsilateral hind paw for up to 28 day. Compared with the MIA and/MIA+Sham Laser groups, seven consecutive days of treatment laser moxibustion at the ST35 increased the PWMT and alleviated the weightbearing difference in the ipsilateral hind paw of KOA rats $(p<0.001)$. Remarkably, on the 17th to 28th day after treatment, the analgesic effect of laser moxibustion on the later stage of pain was obvious, thus suggesting that laser moxibustion may have a certain cumulative therapeutic effect on MIA-induced KOA pain (Fig. 1a, b).

\section{Laser moxibustion prevented articular degradation}

According to the HE and SO staining, both the MIA and MIA+Sham Laser groups exhibited severe cartilage damage and proteoglycan loss 28 days after MIA injection compared with the Saline group. Laser moxibustion significantly inhibited cartilage destruction and proteoglycan loss in MIAinduced KOA rats (Fig. 2a). Consistent with the results of joint staining, a significantly elevated OARSI score was observed in the MIA and MIA+Sham Laser groups compared to the saline group and MIA+Laser group $(p<0.001)$, while the decreased OARSI score in the MIA+Laser group (Fig. 2b) was observed compared to the MIA and MIA+ Sham Laser groups $(p<0.01)$, but its level remained higher than the saline group $(p<0.01)$, indicating that laser moxibustion may have an chondroprotection effect on MIA-induced articular degradation.

\section{Laser moxibustion reduced MMP-13 in the cartilage of MIA-induced KOA}

The expression of MMP-13 in the cartilage was observed through immunohistochemical staining (Fig. 3a). The AOD value of MMP-13 was significantly higher in the MIA and MIA+Sham Laser groups than that in the Saline and MIA+Laser groups $(p<0.01)$. Furthermore, the AOD value of MMP-13 was significantly decreased in laser groups compared with the MIA and MIA+Sham Laser groups $(p<$ $0.01)$, while with no statistical difference compared with saline group $(p>0.05)$. Thus, laser moxibustion treatment inhibited the MMP-13 expression in cartilage of MIAinduced KOA (Fig. 3b).

\section{Laser moxibustion decreased the synovial levels of TNF- $\alpha, I L-1 \beta$, and IL-6 in MIA-induced KOA}

As presented in Fig. $4 \mathrm{a}-\mathrm{c}$, the concentrations of TNF- $\alpha$, IL- $1 \beta$, and IL- 6 in the MIA and MIA+Sham Laser groups were markedly higher than the Saline group and MIA+Laser group $(p<0.05)$. However, the group treated with laser moxibustion had decreased levels of TNF- $\alpha$, IL- $1 \beta$, and IL- 6 compared with the MIA and MIA+Sham Laser groups $(p<0.05)$. There was no statistical difference in the level of these cytokines between the saline and MIA+Laser group $(p>0.05)$. The results suggested that laser moxibustion reduced the expression of proinflammatory cytokines in the MIA-induced KOA model.

\section{Discussion}

Acupuncture and moxibustion has been well demonstrated to relieve joint pain and improves articular dysfunction. ${ }^{12,26}$ However, the adverse effects of moxa smoke restrict the development of traditional moxibustion. ${ }^{27,28}$ In recent years, our group has developed a $10.6 \mu \mathrm{m}$ laser moxibustion apparatus. The infrared radiation spectrum of traditional indirect moxibustion and human acupoints are very similar, and their radiation peaks are about $10 \mu \mathrm{m}$, which may produce resonance, playing a therapeutic role. In this wavelength, the infrared light is easily absorbed by skin, producing a good thermal effect similar to moxibustion. ${ }^{17,18}$ Several clinical trials have shown that low-intensity laser irradiation of corresponding acupoints can significantly alleviate the pain of patients with KOA, promote joint function, and improve the quality of life. ${ }^{13,15,16}$ Basic experimental studies have 

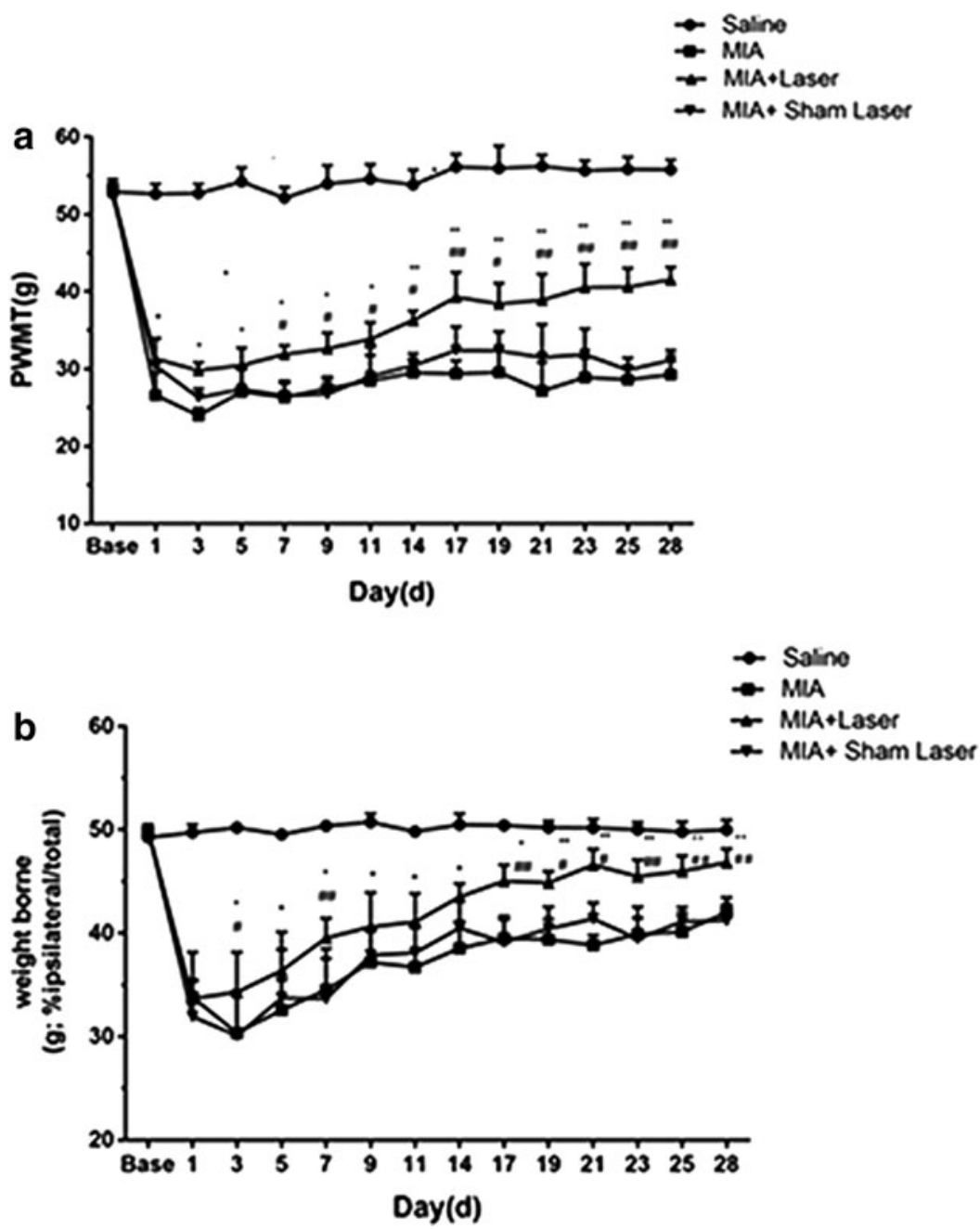

FIG. 1. Effects of laser moxibustion (a) on MIA-induced mechanical hyperalgesia and (b) weight-bearing asymmetry. Data are presented as mean $\pm \operatorname{SD}(n=8)$ and analyzed using two-way ANOVA for repeated measurement. $* p<0.05$ and $* * p<0.001$ compared to MIA group; ${ }^{\#} p<0.05$ and ${ }^{\#} p<$ 0.001 compared to MIA+Sham Laser group. MIA, monosodium iodoacetate; SD, standard deviation. found that a low-intensity laser can inhibit inflammatory cell infiltration, reduce the expression of proinflammatory cytokines and produce antinociceptive effects. ${ }^{29}$ Histopathology showed that a low-intensity laser could alleviate synovitis, cartilage injury, and subchondral bone destruction, which may have a certain cartilage protection effect. ${ }^{30}$ However, most of the currently used low-intensity lasers are semiconductor lasers, and more importantly, they only have the effect of a "light needle" due to their limited thermal effects. ${ }^{31}$ Therefore, they have no significant effect on the treatment of chronic pain. Convincing evidence demonstrated that our $10.6 \mu \mathrm{m}$ laser moxibustion has a long-lasting thermal effect and also affects deep tissues, ${ }^{32}$ which may provide a therapeutic strategy for the clinical treatment of osteoarthritis pain.

MIA is a dose- and time-dependent KOA model. High doses of MIA can induce persistent inflammation, degenerative changes, and chronic pain. ${ }^{3}$ The PWMT and weightbearing difference are the main tests to evaluate the pain behavior of the KOA pain model. ${ }^{33}$ Consistent with other studies, we found that high doses of MIA can induce persistent hyperalgesia and weight-bearing differences. Between day 1 and 7 post-MIA injection was the acute inflammation stage, which caused acute pain. After 14 days, the inflammation was greatly resolved and entered into the stage of chronic pain. Therefore, we applied laser moxibustion in the early time to investigate the role of laser moxibustion on the procession of acute inflammation to chronic pain in the MIA-induced KOA model. The results showed that early laser moxibustion treatment could significantly reverse the PWMT and weight-bearing difference induced by MIA, especially in the later stage of KOA, indicating that laser moxibustion might have a long-lasting antinociceptive effect of MIA-induced chronic pain.

The generation of inflammatory mediators and degradation of the extracellular matrix by MIA-injection are crucial causes for articular cartilage destruction. ${ }^{25}$ In the present study, early laser moxibustion markedly inhibited the destruction of articular cartilage and matrix degradation, and decreased the OARSI score. Furthermore, early laser moxibustion inhibited the increased expression of TNF- $\alpha$, IL-1 $\beta$, IL-6, and MMP-13 in articular cartilage post-MIA injection. Although inflammation gradually dissolved on 14 days, proinflammatory cytokines were still produced, suggesting that MIA-induced KOA models were accompanied by low levels of persistent inflammation during its procession. Overall, our results suggested that the antinociceptive action of $10.6 \mu \mathrm{m}$ laser might occur by inhibiting synovial inflammation, reducing extracellular 
FIG. 2. Effects of laser moxibustion on MIA-induced articular pathological degeneration. (a) The joint histology image of HE and SO staining $(40 \times)$. (b) Histopathologic assessment of cartilage classified using the OARSI scoring system. Data are presented as mean \pm SD $(n=4)$ and analyzed using Kruskal-Wallis test. $* p<0.01$ and $* * p<0.001$ compared to saline group; ${ }^{\# \#} p<0.001$ compared to MIA group; ${ }^{\& \&} p<0.001$ compared to MIA+Sham Laser group. HE, hematoxylin and eosin; OARSI, Osteoarthritis Research Society International; SO, Safranin O.
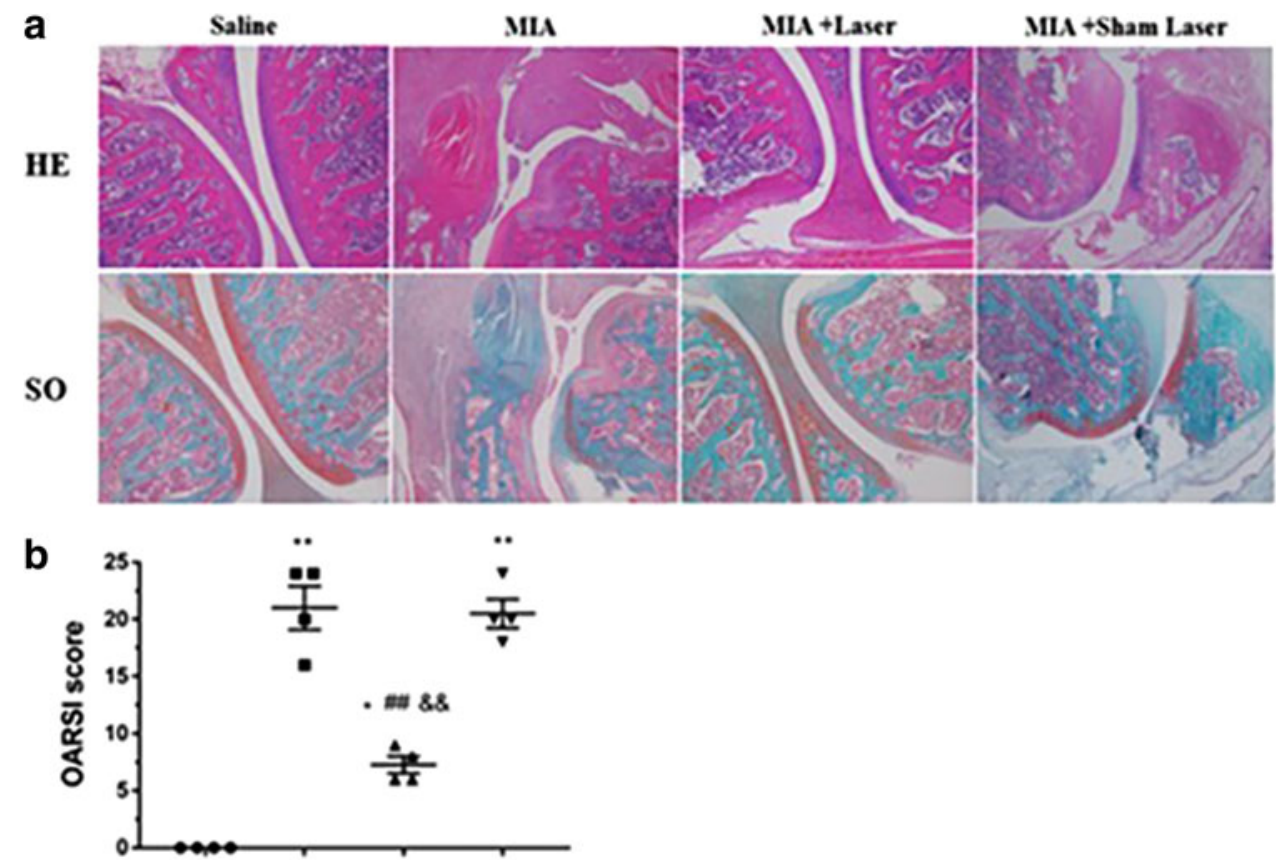

SO
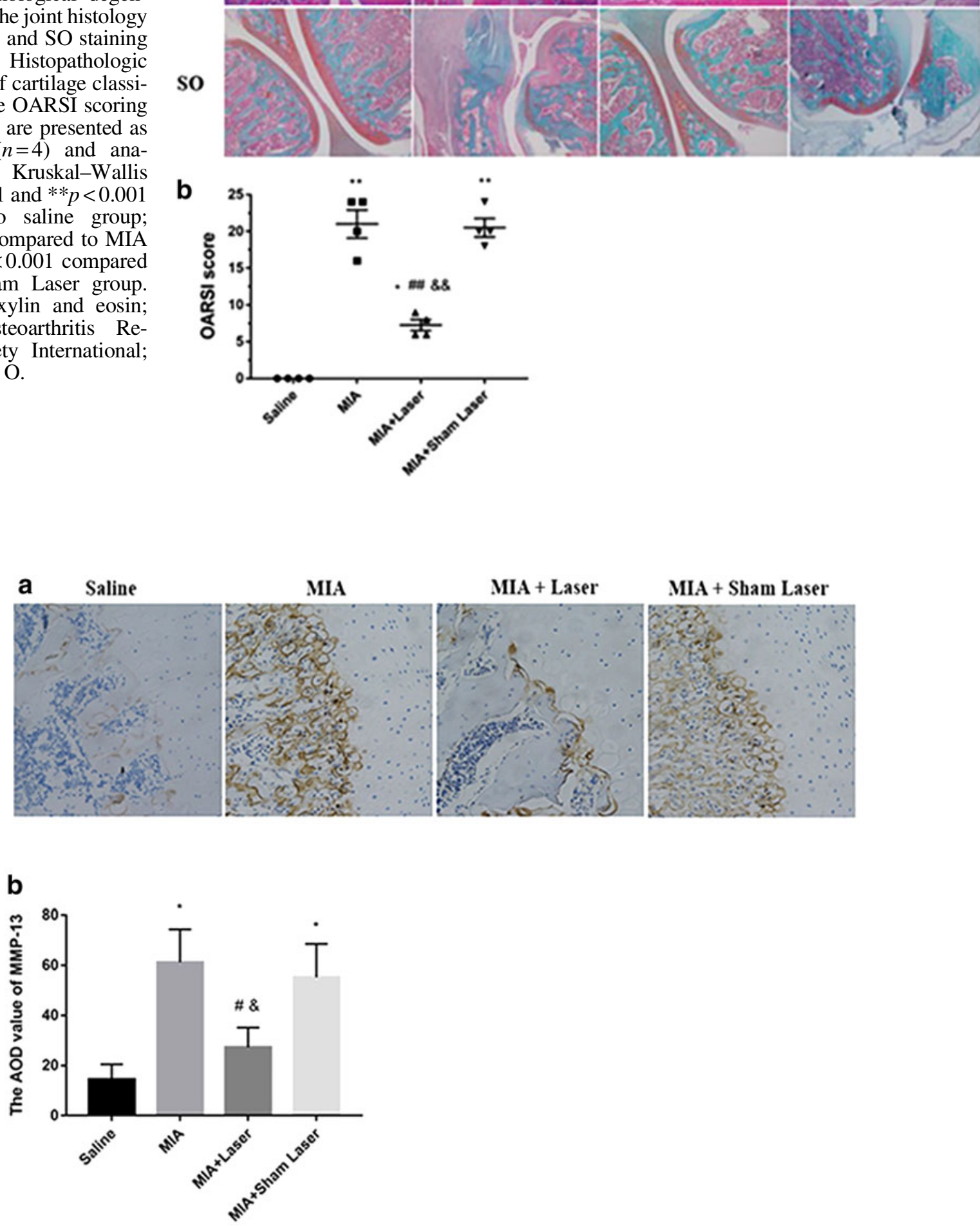

FIG. 3. Effects of laser moxibustion on the expression of MMP-13 in MIA-induced KOA rats. (a) Immunohistochemical staining was used to identify the expression of MMP-13 in the articular cartilage $(200 \times)$. (b) Histogram of the AOD value of MMP-13 expression among the four groups. Data are presented as mean $\pm \mathrm{SD}(n=4)$ and analyzed using one-way ANOVA. ${ }^{*} p<0.01$ compared to saline group; ${ }^{\#} p<0.01$ compared to MIA group; ${ }^{\&} p<0.01$ compared to MIA+Sham Laser group. AOD, average optical density; KOA, knee osteoarthritis; MMP-13, metalloproteinases-13. 

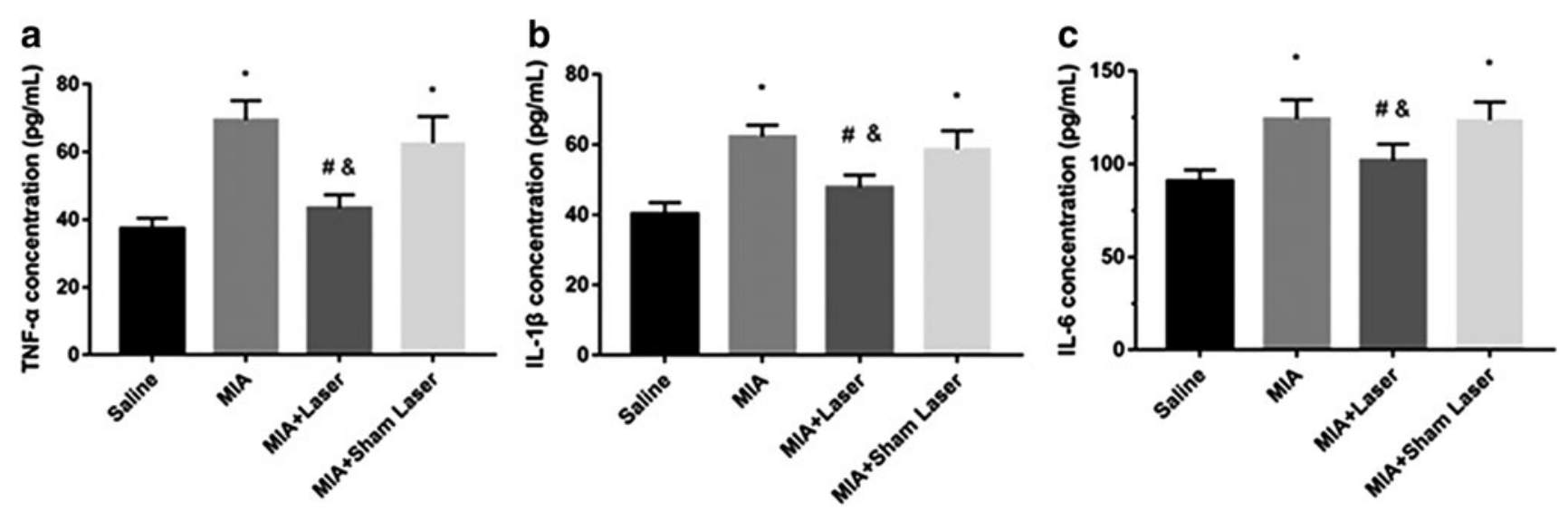

FIG. 4. Effects of laser moxibustion on the level of TNF- $\alpha$, IL- $1 \beta$, and IL- 6 in the synovium of MIA-induced KOA rats. The concentrations of (a) TNF- $\alpha$, (b) IL-1 $\beta$, and (c) IL-6 in the synovium were measured in four groups. Data are presented as mean $\pm \operatorname{SD}(n=4)$ and analyzed using one-way ANOVA. ${ }^{*} p<0.05$ compared to saline group; ${ }^{*} p<0.05$ compared to MIA group; ${ }^{\&} p<0.05$ compared to MIA+Sham Laser group.

matrix catabolism, and preventing cartilage damage, which may underlie the long-lasting analgesia effect mediated by laser moxibustion at the peripheral level.

Chronic pain resulting from osteoarthritis is a serious public health issue. One of the main challenges in chronic pain research is to develop new therapies that are effective for long-term and have fewer side effects. Clinical trials and basic studies have shown that nondrug therapies, such as acupuncture, moxibustion, and low-intensity laser therapy, are important alternative therapies for relieving KOA joint pain and improving joint dysfunction. In this study, we found that $10.6 \mu \mathrm{m}$ laser moxibustion applied in the early stage of MIA-induced KOA significantly inhibit chronic joint pain and joint pathological damage by decreasing levels of TNF- $\alpha$, IL- $1 \beta$, IL- 6 , and MMP-13 in cartilage, which not only induced the chondroprotection effect but also maintained a long-lasting analgesic and antiinflammatory effect. Another study showed that $840 \mathrm{~nm}$ light-emitting diode irradiation for a week after 7 days of MIA-induced KOA prevented cartilage damage and subchondral bone destruction, and significantly reduced inflammatory cell infiltration and pannus formation. ${ }^{34}$ Moreover, Uryu et al. used traditional moxibustion on an injured knee joint and found that there were no antinociceptive effects of moxibustion after 7 days of treatments, while continued moxibustion treatments significantly increased the weight born at day 14 and lasted until the end of day $28 .^{35} \mathrm{Ma}$ et al. also determined that early electroacupuncture (EA) intervention can significantly reduce joint pain and pathological lesions induced by MIA injection compared with mid- and late-term EA interventions. ${ }^{36}$ Interestingly, a study reported that a $808 \mathrm{~nm}$ near infrared laser generated the antianalgesic effect from 5 to $30 \mathrm{~min}$ after treatment and vanished after $60 \mathrm{~min} .{ }^{37} \mathrm{Kim}$ and Kim conducted a 3-week treatment using an $850 \mathrm{~nm}$ galliumaluminum-arsenide laser, resulting in the weight-bearing difference, PWMT, and PWL significantly reversed, and the serum levels of TNF- $\alpha$, IL- $1 \beta$, and IL- 6 also decreased in a MIA-induced KOA model. ${ }^{29}$ These results indicated that different parameters of laser intervention have differ- ent effects on MIA-induced KOA pain. Future studies should compare the parameters of laser, determining the optimal treatment parameters, producing long-lasting analgesic effects, and more importantly, reducing or replacing the use of drugs.

\section{Conclusions and Summary}

This study demonstrated the early intervention of laser moxibustion irradiated at ST35 significantly reversed the PWMT and weight-bearing difference in MIA-induced KOA pain rat. The joint histology showed that $10.6 \mu \mathrm{m}$ laser moxibustion inhibited the procession of cartilage damage and decreased the OARSI score. Moreover, the levels of cartilage MMP-13 and synovial TNF- $\alpha$, IL- $1 \beta$, and IL-6 were also reduced by the early laser moxibustion treatment. These results suggested that $10.6 \mu \mathrm{m}$ laser moxibustion may play an important role in pain relief and chondroprotection in MIA-induced KOA model.

\section{Author Disclosure Statement}

The authors declare that they have no conflict of interest.

\section{Funding Information}

This research was supported by the National Basic Research Program of China (No. 2015CB554505), Shanghai Key Laboratory of Acupuncture Mechanism and Acupoint function (No. 14DZ2260500), Shanghai University of Traditional Chinese Medicine Budget Research Project (No. 18LK010), and Li Yuan's Postgraduate Innovation Ability Project (No. A1-N192050102040105).

\section{References}

1. Hawker GA, Stewart L, French MR, et al. Understanding the pain experience in hip and knee osteoarthritis-an OARSI/OMERACT initiative. Osteoarthritis Cartilage 2008;16:415-422. 
2. McCoy AM. Animal models of osteoarthritis: comparisons and key considerations. Vet Pathol 2015;52:803-818.

3. Udo M, Muneta T, Tsuji K, et al. Monoiodoacetic acid induces arthritis and synovitis in rats in a dose- and timedependent manner: proposed model-specific scoring systems. Osteoarthritis Cartilage 2016;24:1284-1291.

4. Takahashi I, Matsuzaki T, Hoso M. Long-term histopathological developments in knee-joint components in a rat model of osteoarthritis induced by monosodium iodoacetate. J Phys Ther Sci 2017;29:590-597.

5. Zhang RX, Ren K, Dubner R. Osteoarthritis pain mechanisms: basic studies in animal models. Osteoarthritis Cartilage 2013;21:1308-1315.

6. Chen J, Gu YT, Xie JJ, et al. Gastrodin reduces IL-1 $\beta$ induced apoptosis, inflammation, and matrix catabolism in osteoarthritis chondrocytes and attenuates rat cartilage degeneration in vivo. Biomed Pharmacother 2018;97: 642-651.

7. Li Z, Liu B, Zhao D, et al. Omentin-1 prevents cartilage matrix destruction by regulating matrix metalloproteinases. Biomed Pharmacother 2017;92:265-269.

8. Sondergaard BC, Schultz N, Madsen SH, Bay-Jensen AC, Kassem M, Karsdal MA. MAPKs are essential upstream signaling pathways in proteolytic cartilage degradationdivergence in pathways leading to aggrecanase and MMPmediated articular cartilage degradation. Osteoarthritis Cartilage 2010;18:279-288.

9. Mabey T, Honsawek S. Cytokines as biochemical markers for knee osteoarthritis. World J Orthop 2015;6:95-105.

10. Chandran P, Pai M, Blomme EA, Hsieh GC, Decker MW, Honore P. Pharmacological modulation of movementevoked pain in a rat model of osteoarthritis. Eur J Pharmacol 2009;613:39-45.

11. Combe R, Bramwell S, Field MJ. The monosodium iodoacetate model of osteoarthritis: a model of chronic nociceptive pain in rats? Neurosci Lett 2004;370:236-240.

12. Hinman RS, McCrory P, Pirotta M, et al. Acupuncture for chronic knee pain: a randomised clinical trial. JAMA 2014; 312:1313-1322.

13. Helianthi DR, Simadibrata C, Srilestari A, Wahyudi ER, Hidayat R. Pain reduction after laser acupuncture treatment in geriatric patients with knee osteoarthritis: a randomized controlled trial. Acta Med Indones 2016;48: 114-121.

14. Assis L, Milares LP, Almeida T, et al. Aerobic exercise training and low-level laser therapy modulate inflammatory response and degenerative process in an experimental model of knee osteoarthritis in rats. Osteoarthritis Cartilage 2016;24:169-177.

15. Al Rashoud AS, Abboud RJ, Wang W, Wigderowitz C. Efficacy of low-level laser therapy applied at acupuncture points in knee osteoarthritis: a randomised double-blind comparative trial. Physiotherapy 2014;100: 242-248.

16. Mohammed N, Allam H, Elghoroury E, Zikri EN, Helmy GA, Elgendy A. Evaluation of serum beta-endorphin and substance $\mathrm{P}$ in knee osteoarthritis patients treated by laser acupuncture. J Complement Integr Med 2018;15: 20170010.

17. Zhou Y, Shen XY, Ding GH, Deng HP, Zhao L, Lao LX. Infrared radiation spectrum of acupuncture point daling (PC7) on patients with coronary heart disease. Med Acupunct 2009;21:269-274.
18. Shen X, Ding G, Wei J, et al. An infrared radiation study of the biophysical characteristic of traditional moxibustion. Complement Ther Med 2006;14:213-219.

19. Zhao L, Cheng K, Wang L, et al. Effectiveness of moxibustion treatment as adjunctive therapy in osteoarthritis of the knee: a randomized, double-blinded, placebo-controlled clinical trial. Arthritis Res Ther 2014;16:R133.

20. Wu F, Zhao L, Wang L, Zhang H, Lao L, Shen X. Observation on effects of $10.6 \mu \mathrm{m}$ laser moxibustion in patients with knee osteoarthritis: a double-blind, randomized, controlled study. BMC Complement Altern Med 2012;12(Suppl 1):O34.

21. Wu F, Zhang R, Shen X, Lao L. Preliminary study on pain reduction of monosodium iodoacetate-induced knee osteoarthritis in rats by carbon dioxide laser moxibustion. Evid Based Complement Altern Med 2014:754304.

22. Shen D, Wei JZ, Chen LJ, Shen XY, Wang LN. Besides photothermal effects, low-level $\mathrm{CO} 2$ laser irradiation can potentiate skin microcirculation through photobiomodulation mechanisms. Photobiomodul Photomed Laser Surg 2019;37:151-158.

23. Liu L, Zhao L, Cheng K, et al. Examination of the cellular mechanisms of leukocyte elevation by $10.6 \mu \mathrm{m}$ and $650 \mathrm{~nm}$ laser acupuncture-moxibustion. Lasers Med Sci 2018;34: 263-271.

24. Bai F, Ma Y, Guo H, et al. Spinal cord glycine transporter 2 mediates bilateral ST35 acupoints sensitization in rats with knee osteoarthritis. Evid Based Complement Altern Med 2019:7493286.

25. Pritzker KP, Gay S, Jimenez SA, et al. Osteoarthritis cartilage histopathology: grading and staging. Osteoarthritis Cartilage 2006;14:13-29.

26. Song GM, Tian X, Jin YH, et al. Moxibustion is an alternative in treating knee osteoarthritis: the evidence from systematic review and meta-analysis. Medicine (Baltimore) 2016;95:e2790.

27. Kim TH, Kim KH, Kang JW, et al. Moxibustion treatment for knee osteoarthritis: a multi-centre, non-blinded, randomized controlled trial on the effectiveness and safety of the moxibustion treatment versus usual care in knee osteoarthritis patients. PLoS One 2014;9:e101973.

28. Tan C, Wang J, Feng W, Ding W, Wang M. Preliminary correlation between warm needling treatment for knee osteoarthritis of deficiency-cold syndrome and metabolic functional genes and pathways. J Acupunct Meridian Stud 2010;3:173-180.

29. Kim G, Kim E. Anti-inflammation effects of low intensity laser therapy on monosodium iodoacetate-induced osteoarthritis in rats. J Phys Ther Sci 2013;25:173-175.

30. Oliveira P, Santos AA, Rodrigues T, et al. Effects of phototherapy on cartilage structure and inflammatory markers in an experimental model of osteoarthritis. J Biomed Opt 2013; 18:128004.

31. Baxter GD, Bleakley C, McDonough S. Clinical effectiveness of laser acupuncture: a systematic review. J Acupunct Meridian Stud 2008;1:65-82.

32. Wang L, Wu F, Zhao L, et al. Patterns of traditional Chinese medicine diagnosis in thermal laser acupuncture treatment of knee osteoarthritis. Evid Based Complement Altern Med 2013;2013:870305.

33. Suokas AK, Walsh DA, McWilliams DF, et al. Quantitative sensory testing in painful osteoarthritis: a system- 
atic review and meta-analysis. Osteoarthritis Cartilage 2012;20:1075-1085.

34. Jekal SJ, Kwon PS, Kim JK, Lee JH. Effect of $840 \mathrm{~nm}$ light-emitting diode (LED) irradiation on monosodium iodoacetate-induced osteoarthritis in rats. Korean Soc Phys Med 2014;9:151-159.

35. Uryu N, Okada K, Kawakita K. Analgesic effects of indirect moxibustion on an experimental rat model of osteoarthritis in the knee. Acpunct Med 2007;25: 175-183.

36. Ma Y, Guo H, Bai F, et al. A rat model of knee osteoarthritis suitable for electroacupuncture study. Exp Anim 2018;67:271-280.

37. Micheli L, Di Cesare Mannelli L, Lucarini E, et al. Photobiomodulation therapy by NIR laser in persistent pain: an analytical study in the rat. Lasers Med Sci 2017;32: 1835-1846.
Address correspondence to: Xueyong Shen, MD School of Acupuncture-Moxibustion and Tuina Shanghai University of Traditional Chinese Medicine 9415 Room 1200 Cailun Road Shanghai 201203

China

E-mail: snowysh@hotmail.com

Received: June 26, 2019. Accepted after revision: July 25, 2019. Published online: September 24, 2019. 